\title{
Introducing a special issue of the Journal of Muscle Research and Cell Motility on actin and actin-binding proteins
}

\author{
William Lehman ${ }^{1} \cdot$ Yuichiro Maéda ${ }^{2}$
}

Published online: 21 December 2019

C) Springer Nature Switzerland AG 2019

The 47th annual European Muscle Conference was held at the Basic Medical Science Center of Semmelweis University in beautiful and historic Budapest. One session of the conference was devoted to "Actin and Actin-Binding Proteins". What better place to hold this session than in "Albert SzentGyörgyi Hall" at Semmelweis. After all it was in the SzentGyörgyi laboratory in Szeged (Fig. 1) that Straub and Banga first isolated, purified and characterized muscle actin some 75 years prior to the 2018 EMC gathering. Of course, over the ensuing years much has been learned about the ubiquity and multi-faceted roles played by actin in eukaryotic cells. We now know for example that the assembly, structure and function of actin filaments together with the corresponding networks they form are enforced largely by tropomyosin, the actin filament gatekeeper (Dominguez 2011), along with a multitude of actin-binding accessory proteins (Gunning and Hardeman 2017). These proteins and their characteristics were the topic of the session.

Given the current interest in actin-based thin filament structure and function, this journal's editors recommended a special issue dedicated to original research and review articles on topics discussed during the Actin and Actin-Binding Proteins session. This is in keeping with a Journal of Muscle Research and Cell Motility goal to serve it readers by promoting robust discussion of the state of the art research on muscle and cell motility. Fortunately, many of the session's participants agreed to contribute to the Special Issue while also suggesting colleagues to cover additional topics and broaden the dialog.

William Lehman

wlehman@bu.edu

1 Department of Physiology \& Biophysics, Boston University School of Medicine, Boston, MA 02118, USA

2 Structural Biology Research Center, Graduate School of Science, Nagoya University, Furo-cho, Chikusa-ku, Nagoya 464-8601, Japan
Actin has proven to be a very talented and versatile biological actor, always in search of new roles to play inside cells. However, Gunning and Hardeman (2017) and others have pointed out that actin filaments do not generally perform solo, free of cellular partners (also see Manstein et al. in this issue). By analogy to theatrical actors, actin rarely writes its own script or handles its business affairs alone. Instead like most actors, it acquires an agent, in this case tropomyosin, to arrange advantageous roles to perform and well-suited locations in which to act. In fact, most actin filaments in cells are found in association with one or another of 40 or so tropomyosin isoforms that form continuous cables wound along the helically arrayed actin subunits comprising the filament core. The "gatekeeping" agent, tropomyosin, also moonlights as a stage manager giving stability to filament sets and determining which of the plethora of actin-binding proteins to embrace. It is these actin-binding proteins linked to actin that then write out the play's script of actions for the filaments to perform, namely whether to sponsor myosin-based motility or to model and remodel a wide variety of super-molecular cytoskeletal structures. These theatrics are discussed in a diverse group of papers in the Special Issue of the Journal.

Among the submissions, Bugyi and Kellermayer provide a fascinating account of discovery by Banga and Straub, as they first identified and purified actin in the Szent-Györgyi laboratory. Given current trends in protein expression, the paper should be particularly instructive to young biologists, unaccustomed to biochemical procedures of old, and who may never have isolated proteins from tissue or assessed purity without the aid of SDS-PAGE.

Papers that follow by Lehman, Moraczewska and Kopylova et al. characterize tropomyosin and its role in actinbased muscle thin filaments. The structure and function of troponin and leiomodin on thin filaments are discussed by Marston and Zamora and by Moraczewska. Multifaceted myosin-binding protein $\mathrm{C}$ that influences both thin and thick filaments is reviewed by Heling et al. Reviews are also 
Fig. 1 Group portrait of the staff of the Institute of Medical Chemistry and the Institute of Organic and Pharmaceutical Chemistry, Szeged (1938). The photo shows, among others, Brunó F. Straub (left), Ilona Banga (second woman from left), Albert Szent-Györgyi (center) assembled together, at the time their research on muscle proteins began. Reprinted from SZTE info with permission (see https://u-szeged.hu/newsand-events/2017/albert-szent -gyorgyi-was?objectParentFol derId=26342)

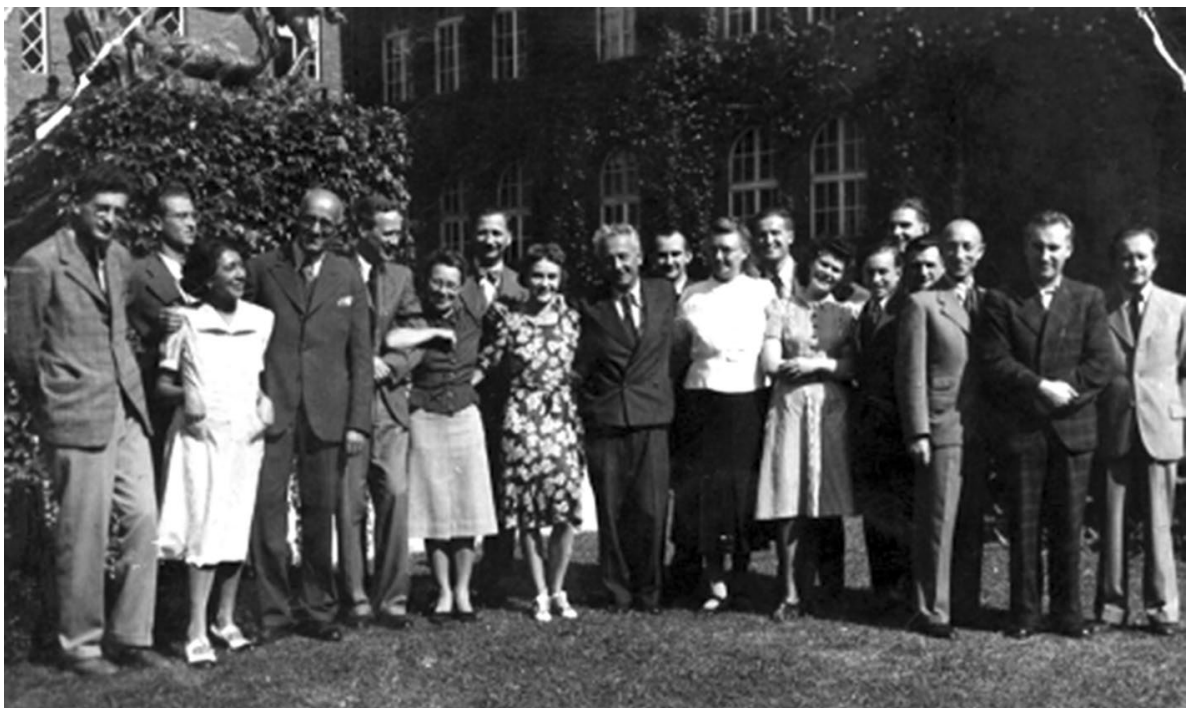

provided on the structural and functional impact of two giant muscle proteins nebulin (by Yuen and Ottenheijm) and titin (by Nishikawa et al.). Actin-binding proteins such as fragmin and cofilin are highlighted (by Takeda et al. and by Narita), as are striking cell imaging and biophysics of actin dynamics (by Yamashiro and Watanabe and by Romet-Lemonne and Jégou).

The Special Issue was not intended to be a comprehensive monograph or the final word on actin's functional interactions. (Wikipedia currently lists a cast of more than 250 actin-binding proteins [shttps://en.wikipedia.org/wiki/Actin -binding_protein].) In particular, it would be appropriate for future issues of the journal to cover current work on other actin-binding proteins, for example $\alpha$-actinin and filamin, that are abundant both in muscle and non-muscle cells. Actin interactions with the Z-disc interactome in striated muscle or analogously with dense bodies and focal adhesion structures in smooth muscle and non-muscle cells would also make useful topics to review.

\section{References}

Dominguez R (2011) Tropomyosin: the gatekeeper's view of the actin filament revealed. Biophys J 100:797-798

Gunning PW, Hardeman EC (2017) Tropomyosins. Curr Biol 27:R8-R13

Publisher's Note Springer Nature remains neutral with regard to jurisdictional claims in published maps and institutional affiliations. 